\title{
The Effect of Management Strategy of ST Segment Elevation Myocardial infarction in Elderly on LV remodeling and Clinical Outcome
}

O.S.Arafa' S.A.Mostafa, A.S.M.Sabry ,S.I. Farag and S. M. Ahmed

Cardiology Dept., Faculty of Medicine, Benha Univ.,Benha, Egypt

E-Mail:Shereenmostafa646@gmail.com

\begin{abstract}
Acute ST-segment elevation myocardial infarction (STEMI) is the acute injury may evolve in the left ventricular remodeling despite effective coronary revascularization and optimal medical therapy and leads to advanced heart failure. The aim of the present study was to assess the effect of management strategy of ST segment elevation myocardial infarction in elderly on LV remodeling and clinical outcome.This study included 150 elderly patients above 60 years old with ST segment elevation myocardial infarction referred to the Coronary care unit, Benha University Hospital.They classified into 3 groups:patients who treated with conservative therapy(Group I),patients who treated with primary percutaneous intervention(Group II) and patients who treated with fibrinolytic therapy(Group III). Standard two-dimensional echocardiography,TDI derived velocity and speckle tracking and 3D echocardiography were used to assess LV remodeling and clinical outcome.Patients of group I were significantly older than those of group II and group III( $p$ value $<0.001)$. Male gender was more prevalent in patients of 3 groups ( $\mathrm{P}$ value $<0.001$ ).Early diastolic mitral annular velocity $(\mathrm{e} ")$ was significantly reduced in patients of group I and group II compared with patients of group III(p value $<0.001)$ and the mitral E/e" ratio was significantly higher in patients of group I and group II compared with patients of group III ( $p$ value $<0.001$ ). There was no difference in management strategy of ST myocardial infarction patients as regarding conservative therapy, primary coronary intervention and fibrinolytic therapy.
\end{abstract}

Keywords: Primary coronary intervention, LV remodeling, Fibrinolytic therapy, Conservative therapy.

\section{Introduction}

It is known that in patients who experienced acute STsegment elevation myocardial infarction (STEMI), the acute injury may evolve in the left ventricular remodeling (LVR) despite effective coronary revascularization and optimal medical therapy. Predicting LVR in these patients can be challenging [1].

LV remodeling is an important factor in the pathophysiology of advancing heart failure (HF) and several studies support the role of measures of LV remodeling in the clinical investigation of novel HF treatments [2].

Postinfarct ventricular remodelling develops in about 30\% patients with a history of myocardial infarction [3] .As remodelling depends on infarct size [4], it is likely that its prevalence is higher in the subgroup of patients without any or successful reperfusion.

The age of patients suffered from ST-elevation myocardial infarction (STEMI) is expected to rise in the next decades. In elderly patients, the outcome after STEMI is worse than in younger people, but they are less likely to receive reperfusion therapies [5].

As fibrinolytic therapy could be associated with increased bleeding risk, especially in the elderly, the advantages of primary percutaneous coronary intervention (PPCI) in elderly patients with acute STEMI are apparent[6].Emerging echocardiographic techniques, STE; strain \& strain-rate imaging has gained acceptance for its usefulness in defining the degree of myocardial

deformation and contractility in patients with various cardiac diseases [7].

\section{Patients and methods}

This study included 150 elderly patients above 60 years old with ST segment elevation myocardial infarction referred to the Coronary care unit, Benha University
Hospital. They classified into 3 groups: patients who treated with conservative therapy(Group I).patients who treated with primary percutaneous intervention(Group II).patients who treated with fibrinolytic therapy(Group III).It was conducted at coronary care unit at "Benha University hospital" during the period from March 2018 to September 2019.

Consent from the patients and the approval from the ethical committee was obtained.

Patients with primary valvular disease, congenital heart disease, prior myocardial infarction, previous history of primary coronary intervention (PCI) or coronary artery bypass graft $(\mathrm{CABG})$, rhythm other than sinus, poorly echogenic patients and patients refusing to participate in this study were excluded.

The following data were collected

Detailed history taking name, age, sex, occupation, special habits, risk factors for ischemic heart disease, time of chest pain.

Full clinical examination

Electrocardiography: Twelve lead surface ECG was done for each patient to detect presence or absence of ST segment deviation.

\section{Echocardiography}

Comprehensive transthoracic echocardiographic examinations were performed "before discharge and 3 months after discharge", using a Philips, Epic 7C machine, with the $5.5 \mathrm{X}$ transducer $\mathrm{S} 5-1$ probe with simultaneous ECG signal. Patients were examined in the left lateral decubitus position. All echocardiographic examinations were obtained and recorded offline.

A) Two-dimensional transthoracic echocardiography (TTE); was used to assess mitral regurgitation,LVM and LVMI. 
B) Tissue Doppler Imaging: The pulsed wave TDI sample volume was placed on the mitral annulus in the apical four- and two-chamber views.Pulsed wave tissue Doppler imaging (TDI) was performed in the apical views to acquire mitral annular velocities. Measurements included the systolic (S) and early diastolic ( $\left.E^{\prime}\right)$.

C) Speckle tracking echocardiography (STE): was performed in all patients with ST segment myocardial infarction to assess myocardial deformation in apical -4 chamber, apical -2 chamber and apical long axis views.

The global longitudinal strain (GLS) will be determined by averaging all peak systolic segmental strain values from the 3 standard apical views. Longitudinal peak strain values were averaged over 2 consecutive cardiac cycle.

\section{D) 3D echocardiography}

\section{- LV Volumes and LV ejection fraction:}

After manual identification of the mitral valve plane and the apex with 2 reference points on the end diastolic and end systolic frames, the software identifies the endocardial border in each frame, an LV 3D model will be generated and LV volumes and LVEF will be calculated.

\section{- Left ventricular dyssynchrony:}

The systolic dyssynchrony index (percentage of cardiac cycle) from 16 and $12 \mathrm{LV}$ segments on real time 3D echocardiography was analyzed to calculate LV dyssynchrony (defined as standard deviation of the time to search minimum systolic volume for $16 \mathrm{LV}$ segments) according to a17 segment model.

In hospital outcome according to clinical, angiographic and procedure success.

Three months follow up:

A. Major adverse cardiovascular events:

1. Recurrent chest pain

2. Recurrent infarction

3. Mortality

4. Heart failure

5. Stroke

6. Arrhythmia

7. Complications as bleeding and cardiogenic shock.

8. Other cause mortality
B.Echocardiography: the previous echocardiographic parameters were reassessed again.

\section{Statistical analysis of the data}

Data were fed to the computer using IBM SPSS software package version 25.0.

Qualitative data were described using number and percent. Comparison between different groups regarding categorical variables was tested using Chi-square test.

\section{Results and discussion}

There is very limited evidence in the recommendation of the reperfusion strategy for elderly STEMI patients $\{8\}$.Age is an independent predictor of adverse outcomes in the elderly, so older patients are in accordance at higher risk of death compared with the younger patients [9].

Immediate reperfusion was proved to be beneficial in younger STEMI patients ( $<75$ years old) in previous studies. However, older patients were usually excluded, especially in the randomized trials [10]. Older people were more likely to contract MI complications than the younger patients because of the reduced heart function, and the degraded heart structure [10].

Older people were also at higher risk of hemorrhage stroke when they had fibrinolysis, or of gastrointestinal bleeding when they were under dual antiplatelet or triple anticoagulation therapy [11].

Older people properly had worse renal function than the younger ones, so the contrast used in primary PCI might elevate the risk of renal failure and contrast-induced nephropathy [12].

Lack of sufficient evidence, it is still unclear if immediate reperfusion was effective and safe in the elderly, or what immediate reperfusion was best, as well. Left ventricular remodeling is disadvantageous process resulting in progressive enlargement and changing the shape of left ventricle cavity with systolic dysfunction as an organ adaptation to the new conditions associated with tissue infarction [13].

The phenomenon is complex, related to macro- and microscopic changes in the structure and function of cardiomyocytes. Left ventricular remodeling is well evidenced to be associated with worse outcomes and to predispose to heart failure and death [14], and for this reason it assumes a negative prognostic value [15].

Table (1) Comparison between studied groups regarding age and sex

\begin{tabular}{|c|c|c|c|c|c|c|c|}
\hline & \multicolumn{2}{|c|}{ Group I } & \multicolumn{2}{|c|}{ Group II } & \multicolumn{2}{|c|}{ Group III } & \multirow[t]{2}{*}{$P$ value } \\
\hline & No & $\%$ & No & $\%$ & No & $\%$ & \\
\hline Sex & & & & & & & $<0.001$ \\
\hline Male & 27 & 60 & 7 & 63.6 & 84 & 89.4 & \\
\hline Female & 18 & 40 & 4 & 36.4 & 10 & 10.6 & \\
\hline Age ( Mean + SD $)$ & $73 \pm 9$ & & & & $65 \pm 5$ & & $<0.001$ \\
\hline
\end{tabular}

Kruskal Wallis test was used for numerical data. Post hoc was done in case of significant overall effect. Different letters indicate significant pairs.

Categorical data was compared using Chi-square test. 
Table (2) Comparison between studied groups regarding risk factors

\begin{tabular}{lccccccc}
\hline & \multicolumn{2}{c}{ Group I } & \multicolumn{2}{c}{ Group II } & \multicolumn{2}{c}{ Group III } & \multicolumn{1}{c}{ P } \\
\cline { 2 - 7 } & No & \% & No & \% & No & \% & value \\
\cline { 1 - 6 } DM & 22 & 48.9 & 4 & 36.4 & 23 & 24.5 & 0.016 \\
HTN & 18 & 40.0 & 2 & 18.2 & 25 & 26.6 & 0.183 \\
Smoking & 15 & 33.3 & 6 & 54.5 & 60 & 63.8 & 0.003 \\
Dyslipidemia & 32 & 71.1 & 5 & 45.5 & 54 & 57.4 & 0.171 \\
Family H/O & 11 & 24.4 & 5 & 45.5 & 23 & 24.5 & 0.311 \\
\hline
\end{tabular}

Kruskal Wallis test was used for numerical data. Post hoc was done in case of significant overall effect. Different letters indicate significant pairs. Categorical data was compared using Chi-square test.

Table (3 ) In-hospital complications in patients of studied groups

\begin{tabular}{lccccccc}
\hline & \multicolumn{2}{c}{ Group I } & \multicolumn{2}{c}{ Group II } & \multicolumn{2}{c}{ Group III } & \multicolumn{1}{c}{ P } \\
\cline { 2 - 6 } & No & $\mathbf{\%}$ & No & $\mathbf{\%}$ & No & \% & value \\
\hline Re infarction & 3 & 6.7 & 0 & 0 & 7 & 7.4 & 1.0 \\
HF & 22 & 48.9 & 9 & 81.8 & 60 & 63.8 & 0.079 \\
Mortality & 1 & 2.2 & 1 & 9.1 & 4 & 4.3 & 0.483 \\
Arrhythmia & 21 & 46.7 & 5 & 45.5 & 60 & 63.8 & 0.114 \\
Stroke & 5 & 11.1 & 2 & 18.2 & 12 & 12.8 & 0.818 \\
Bleeding & 5 & 11.1 & 1 & 9.1 & 9 & 9.6 & 0.956 \\
Cardiogenic & 7 & 15.6 & 5 & 45.5 & 13 & 13.8 & 0.028 \\
shock & & & & & & & \\
\hline
\end{tabular}

Chi-square or Fisher's exact test was used

In the current study, Patients of group I were significantly older than those of group II and group III ( $73 \pm 9$ vs. $66 \pm 7$ vs. $65 \pm 5$ years, $P$ value $<0.001)$ Table (1).

This was similar to Peiyuan et al. who studied The Comparison of the Outcomes between Primary PCI, Fibrinolysis and No Reperfusion in Patients $\geq 75$ Years Old with ST-Segment Elevation Myocardial Infarction. They found patients who had conservative therapy were relevantly older than patients with PCI nor patients with fibrinolytic therapy $(80.59 \pm 4.57$ vs. $79.59 \pm 4.04$ vs. $78.79 \pm 7.12$ years, $p$ value $<0.001)[16]$.

In contrast to Alkhushail et al. who studied Prognosis of primary percutaneous coronary intervention in elderly patients with ST-elevation myocardial infarction. They found that there was no statistical difference regarding to age between patients with PCI and patients who had conservative therapy (83.92 vs. 84.76 years, $p$ value $>0.05$ ) [17].

According to $\{18\}$ who studied Primary angioplasty vs. fibrinolysis in very old patients with acute myocardial infarction: TRIANA (TRatamiento del Infarto Agudo de miocardio eN Ancianos) randomized trial and pooled analysis with previous studies. They found that there was no statistical difference regarding to age between patients with PCI and patients who had fibrinolytic therapy $(81.2 \pm 4.6$ vs. $81 \pm 4.3$ years, $p$ value $>0.05$ ).

This could be explained by the presence of other comorbidities as diabetes, hypertension, heart

failure and many risk factors in patients who had conservative therapy in the present study.

In the current study, Male gender was more prevalent in patients of 3 groups ( $\mathrm{P}$ value $<0.001$ ). Group I patients had 27 males $(60 \%)$ and 18 females (40\%), group II had 7 males
(63.6\%) and 4 females (36.4\%),group III had 84 males(89.4\%) and 10 females(10.6\%) Table(1).

This was similar to $\{16\}$ who found that Men were more likely to have primary PCI $(64.3 \%)$ or

fibrinolysis $(62.5 \%)$ and conservative therapy (56\%) compared with women ( $\mathrm{p}$ value $<0.001$ ).

In contrast to $\{17\}$ found that there was no statistical difference regarding to sex between patients with PCI and patients who had conservative therapy ( $p$ value $>0.05$ ). Patients with PCI had 80 males(43\%) and 106 females(57\%) while patients with conservative therapy had 27 males(52\%) and 25 females $(48 \%)$.

Also, $\{18\}$ reported that there was no statistical difference regarding to sex between patients with PCI and patients who had fibrinolytic therapy ( $p$ value $>0.09$ ).

This could be explained by the difference in clinical characteristics between the studied groups.

In the present study, there were no differences observed between the 3groups as regard to other risk factors: DM, HTN, smoking, dyslipidemia and family history of CAD(pvalue $>0.05)$ (table2). This was similar to[16]\} who found that there was no statistical difference regarding risk factors between patients with PCI, patients with fibrinolysis and with conservative therapy except dyslipidemia was significantly higher in patients with PCI ( $\mathrm{p}$ value $<0.001$ ).

In contrast to [17] who found that risk factors were significantly higher in patients with conservative therapy than patients with PCI ( $p$ value $<0.001)$.

In the current study, there was no significant statistical difference between the three groups as regarding in hospital complications as HF, cardiogenic shock, minor bleeding, reinfarction, mortality, stroke and arrhythmia Table (3). 
This was consistent with $\{16\}$ who found that reinfarction,stroke, arrhythmia and minor bleeding were similar between PCI, fibrinolytic and conservative therapy patients( $\mathrm{p}$ value $>0.05)$, HF and mortality were significantly lower in PCI patients compared with fibrinolytic and conservative patients ( $\mathrm{p}$ value $<0.05$ ).

This was similar to $\{18\}$ who found there was no statistical difference between patients with PCI and fibrinolytic therapy regarding in hospital complications as $\mathrm{HF}$, mortality, cardiogenic shock, stroke, bleeding and reinfarction ( $p$ value $>0.05$ ).

\section{Conclusion}

There was no difference in management strategy of ST myocardial infarction patients as regarding conservative therapy, primary coronary intervention and fibrinolytic therapy.

\section{References}

[1] A.Galli, A.Lombardi, Postinfarct left ventricular remodelling: a prevailing cause of heart failure. Cardiology research and practice, 2016.

[2] M.A.Konstam, D.G.Kramer, A.R.Patel, M.S.Maron, J.E.Udelson. Left ventricular remodeling in heart failure: current concepts in clinical significance and assessment. JACC: Cardiovascular Imaging , Vol. 4, PP.98-108,2011.

[3] F.A.Flachskampf, M.Schmid, C.Rost, S.Achenbach, A.N. DeMaria, W.G. Daniel. Cardiac imaging after myocardial infarction. European heart journal, Vol.32, PP.272283,2010 .

[4] T.Springeling, S.W.Kirschbaum, A.Rossi, T.Baks, Y. Karamermer, A.Schulz, et al. Late cardiac remodeling after primary percutaneous coronary intervention. Circulation Journal, Vol. 77,PP. 81-88,2013.

[5] J. Xiao, B.Shen, J.Li, D.Lv, Y.Zhao, F.Wang, et al. Serum microRNA-499 and microRNA-208a as biomarkers of acute myocardial infarction. International journal of clinical and experimental medicine,Vol. 7, PP.136,2014.

[6] S.J.Chen, Z. Gong, Q.L. Duan. Evaluation of heart function with impedance cardiography in acute myocardial infarction patients. International journal of clinical and experimental medicine,Vol. 7, PP.719,2014.

[7] M.Leitman, P.Lysyansky, S. Sidenko, V.Shir, E.Peleg, M.Binenbaum, et al. Two-dimensional strain-a novel software for real-time quantitative echocardiographic assessment of myocardial function. Journal of the American Society of Echocardiography ,Vol. 17,PP. 1021-1029,2004.

[8] R.J.Shelton, A.M.Crean, K.Somers, C.Priestley, C.Hague, J.M.Blaxill, et al. Real-world outcome from ST elevation myocardial infarction in the very elderly before and after the introduction of a 24/7 primary percutaneous coronary intervention service. American heart journal, Vol. 159,PP. 956-963,2010.
[9] V.Oduncu, A.Erkol, A.C.Tanalp, C.Kırma, M.Bulut, A.Bitigen, et al.Comparison of early and late clinical outcomes in patients $\geq 80$ versus $<80$ years of age after successful primary angioplasty for ST segment elevation myocardial infarction. Archives of the Turkish Society of Cardiology, Vol.41,PP. 319-328,2013.

[10] L.Antonsen, L.O.Jensen, C.J.Terkelsen, H.H.Tilsted, A.Junker, M.Maeng, et al. Outcomes after primary percutaneous coronary intervention in octogenarians and nonagenarians with ST-segment elevation myocardial infarction: from the Western Denmark heart registry. Catheterization and cardiovascular interventions, Vol.81,PP. 912-919,2013.

[11] N.S.Abraham, C. Hartman, P.Richardson, D.Castillo, J.R.L.Street Jr,et al. Risk of lower and upper gastrointestinal bleeding, transfusions, and hospitalizations with complex antithrombotic therapy in elderlypatients. Circulation,Vol.128,PP.1869-1877,2013.

[12] N. Ruparelia , A. Chieffo . Dual antiplatelet therapy following drug-eluting stent implantation: how long is long enough? Expert Rev Cardiovasc Ther,Vol.13,PP.585-587,2015.

[13] A.J.McLellan, M.P. Schlaich, A.J.Taylor, S.Prabhu, D.Hering, L.Hammond,et al. Reverse cardiac remodeling after renal denervation: atrial electrophysiologic and structural changes associated with blood pressure lowering. Heart Rhythm.Vol.12,PP.982-990,2015.

[14] J.Y. Jang, J.S. Woo, W.S. Kim, S.J. Ha, I.S.Sohn, W.Kim,et al. Serial assessment of left ventricular remodeling by measurement of left ventricular torsion using speckle tracking echocardiography in patients with acute myocardial infarction. The american journal of cardiology,Vol. 106(7),PP. 917-923,2010.

[15] M.A.Konstam, D.G.Kramer, A.P.Patel, M.S.Maron, J.E.Udelson. Left ventricular remodeling in heart failure: current concepts in clinical significance and assessment. JACC:Cardiovascular Imaging,Vol. 4(1), PP.98-108,2011.

[16] H.Peiyuan, Y. Jingang, X.Haiyan, G. Xiaojin, X. Ying, W.Yuan, et al. The comparison of the outcomes between primary PCI, fibrinolysis, and no reperfusion in patients $\geq$ 75 years old with ST-segment elevation myocardial infarction: results from the Chinese Acute Myocardial Infarction (CAMI) Registry. PloS one, Vol. 11(11),PP. e0165672,2016.

[17] A.Alkhushail, S.Kohli, A.Mitchel, R.Smith, C.Ilsely. Prognosis of primary percutaneous coronary intervention in elderly patients with ST-elevation myocardial infarction. Journal of the Saudi Heart Association,Vol. 27(2),PP.85-90,2015.

[18] H.Bueno, A.Betriu, M.Heras, J.J.Alonso, A.Cequier, E.J.García, et al. Primary angioplasty vs. fibrinolysis in very old patients with acute myocardial infarction: TRIANA (TRatamiento del Infarto Agudo de miocardio eN Ancianos) randomized trial and pooled analysis with previous studies. European heart journal, Vol.32(1),PP. $51-60,2010$. 